\section{EFFECTIVE PROTECTION FOR VULNERABLE DECIDUOUS TEETH}

GC's Fuji Triage is a simple yet effective treatment for vulnerable teeth in need of protection.

Children with fragile, erupting teeth need protection fast. Effective protection is essential to prevent the onset of early decay. Fuji Triage already has a strong track record of preventing caries in erupting teeth. This self-adhesive material is insensitive to moisture and has a low enough viscosity to penetrate pits and fissures, making it truly effective.

With a very high level of fluoride release which is six times higher than any other glass ionomer material, Fuji Triage can be applied when saliva control is not possible - particularly useful when treating young children. Due to the inherent chemical bonding of Fuji Triage this material chemically bonds to the tooth structure without the need to etch or use a separate bonding agent.

Fuji Triage is a self-curing glass ionomer cement with an optional command set if access allows. The unique pink colour

absorbs the heat from the curing light to speed up the setting as well as aiding

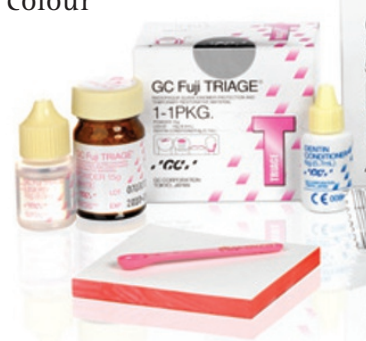

visualisation and monitoring, so you know exactly where you have placed the material.

For further information please contact GC UK on 01908218999.

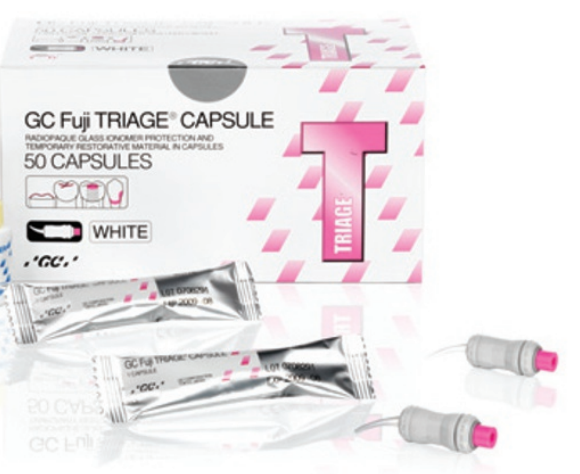

\title{
DENTISTS CREATE GUIDE TO BUYING YOUR FIRST PRACTICE
}

Dentists Saeid Haghri and Sundeep Rooprai have created Fire Ant Assessment - a tool to help other dentists carry out a comprehensive assessment of a dental practice (business) they are considering buying.

Saeid and Sundeep found it very difficult to assess a dental practice for purchase for the first time; they had no idea where to start and there did not seem to be any clear step-by-step instructions explaining how to approach the process. Having designed their own practice selection criteria, the pair decided to turn them into a comprehensive booklet.

The Fire Ant Assessment is intended to help dentists make one of the most important decisions in their life: the purchase of a dental practice.

This educational tool for first time buyers is offered to dentists for free at www.fireantassessment. com along with a user guide video.

Funds contributed by sponsors and individuals will go to the Make a Dentist (MAD) charity. MAD was set up in 2009 by dental students from the University of London and raises money for dental students in Zimbabwe.

\section{NEW IMPLANT SOFTWARE TO MEET ALL YOUR NEEDS}

3Shape, who make 3D scanners and CAD/CAM software solutions, has released its new Implant Studio software to the European market. The solution is designed for use in both clinics and labs, and 3Shape is offering Implant Studio in various configuration packages to match the different needs of both.

You can now look forward to a solution that finally brings together the latest technologies within implant planning into a single smooth workflow. 3Shape's solution includes:

- A complete digital workflow for dentist and labs

- All the restorative components can be provided to the dentists before surgery
- Easy Implant Planning with intuitive tools that merge the benefits of planning in both 3D and 2D

- Virtual Crown functionality - optimal implant placement in combination with the intended prosthetic design

- Surgical Guides - Design cost-efficient surgical guides ready for local manufacturing

- 3Shape Communicate integration - makes it easy to receive 3D surface scans - from TRIOS and from 3shape desktop scanners, and send approved implant positions for designing abutments and crowns in Dental System.

- Open software platform - Implant Studio supports open DICOM CT scans and STL surface scans as well as implant systems (libraries) from major implant manufacturers.

Implant Studio will be available through 3Shape resellers. Contact your supplier or visit www.3shapedental.com.

\section{THE COMPLETE PRACTICE} WORKFLOW, FREE

The first ever free and open source Dental Practice Management Software has been certified by the NHS to transmit claims on behalf of UK practices with an NHS contract.

The new 'Spinnaker' software, created by Derbyshirebased IT specialist Dental IT, helps dental professionals improve everyday performance, create a better patient experience and streamline their time and costs. The ability for Web EDI means that dentists with an NHS contract can transmit their FP17 claims for courses of treatment electronically to the NHS directly from the software without manually completing forms.

Spinnaker enables users to freely develop their own features to match the individual needs and requirements of a particular practice. With the programme currently in the final stages of development and beta testing, Dental IT is looking for dental professionals to test the system.

For more details and to find out how you can get the free software, visit www.dentalit. ltd.uk/spinnaker. 